complexe permet la dégradation par le protéasome d'une partie des protéines NCC produites dans le réticulum endoplasmique. En présence de mutations des gènes KLHL3 ou Cullin-3, I'interaction des protéines avec leur substrat NCC est altérée et la dégradation du cotransporteur via le protéasome est réduite. $\varepsilon$ n conséquence, un plus grand nombre de molécules NCC seraient présentes à la membrane, entraînant l'augmentation de l'activité de réabsorption rénale d'ions $\mathrm{Na}^{+}$et $\mathrm{Cl}^{-}$. Cette activité accrue de NCC rend compte de la grande sensibilité des patients aux diurétiques thiazidiques, NCC étant la cible privilégiée de ces agents pharmacologiques.

\section{Une nouvelle voie de recherche}

De nombreux points restent à élucider, parmi lesquels : existe-t-il d'autres transporteurs cibles de ce système d'ubiquitination? Existe-t-il d'autres protéines Kelch (famille de 38 membres) capables de lier la culline-3 au niveau rénal et de jouer un rôle dans la régulation du transport ionique et de la pression artérielle? Quelles sont les interactions directes et indirectes entre les différents partenaires? Quelles sont les relations avec WNKl et WNK4 dont les mutations sont responsables de la même pathologie? Comment expliquer que des mutations dans des protéines aussi ubiquitaires entraînent un phénotype essentiellement rénal ?

$\varepsilon n$ conclusion, deux études parallèles ont identifié deux gènes inattendus responsables d'une forme mendélienne d'hypertension artérielle. Les protéines correspondantes appartiennent à un mécanisme de dégradation de molécules impliquées dans le transport ionique. Comme cela fut le cas pour les gènes WNKI et WNK4 dix ans auparavant, la découverte de mutations dans des gènes codant pour des acteurs insoupçonnés pose de nombreuses questions physiopathologiques et ouvre une nouvelle voie de recherche. $\checkmark$ KLHL3 and CULLIN-3: new genes involved in familial hypertension

\section{LIENS D'INTÉRÊT}

Les auteurs déclarent n'avoir aucun lien d'intérêt concernant les données publiées dans cet article.

\section{RÉFÉRENCES}

1. Gordon RD. Syndrome of hypertension and hyperkalemia with normal glomerular filtration rate. Hypertension $1986 ; 8$ : 93-102.

2. Wilson FH, Disse-Nicodeme S, Choate KA, et al. Human hypertension caused by mutations in WNK kinase. Science 2001 ; 293 : 1107-12.

3. Hadchouel J, Delaloy C, Jeunemaitre X. WNKI et WNK4, nouveaux acteurs de l'homéostasie hydrosodée. Med Sci (Paris) $2005 ; 21: 55-60$.

4. Hadchouel J, Jeunemaitre X. Life and death of the distal nephron: WNK4 and NCC as major players. Cell Metab 2006; $4: 335-7$

5. Boyden LM, Choi M, Choate KA, et al. Mutations in kelch-like 3 and cullin 3 cause hypertension and electrolyte abnormalities. Nature 2012 ; 482 : 98-102

6. Louis-Dit-Picard H, Barc J, Trujillano D, et al. KLHL3 mutations cause familial hyperkalemic hypertension by impairing ion transport in the distal nephron. Nat Genet $2012 ; 44:$ 456-60.

7. Wilhelm K. BTB protein as henchmen of Cul3-based ubiquitin ligases. Nat Cell Biol $2003 ; 5: 950-1$.

8. Pintard L, Willems A, Peter M. Cullin-based ubiquitin ligases: Cul3-BTB complexes join the family. EMBO J $2004 ; 23: 1681-7$.

9. Needham PG, Mikoluk K, Dhakarwal P, et al. The thiazide-sensitive $\mathrm{NaCl}$ cotransporter is targeted for chaperone-dependent endoplasmic reticulumassociated degradation. J Biol Chem 2011 ; 286 : 43611-21.

10. Mount DB. Regulated endocytosis of NCC. Am J Physiol Renal Physiol 2010 ; 299 : F297-9.

11. Frindt G, Palmer LG. Surface expression of sodium channels and transporters in rat kidney: effects of dietary sodium. Am J Physiol Renal Physiol 2009 ; 297 F1249-55.

\title{
NOUVELLE
}

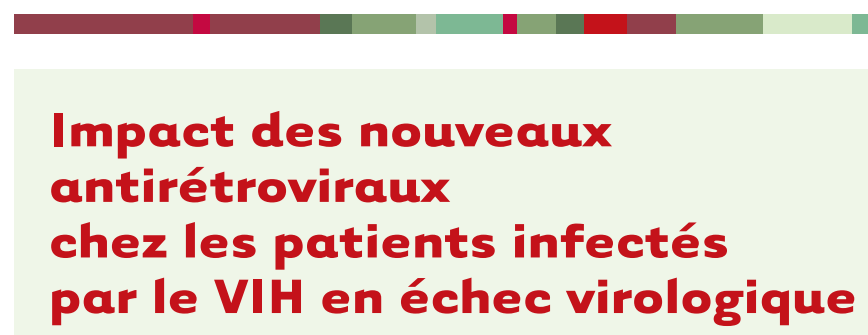

Dominique Costagliola

> Les antirétroviraux, qui permettent de contrôler la charge virale du VIH (virus de l'immunodéficience humaine), sont apparus sur le marché en 1987. II s'agissait d'inhibiteurs nucléosidiques de la transcriptase inverse. Jusqu'en 1996, ils ont été principalement utilisés en mono- ou bithérapie. À partir de 1996, une deuxième famille est apparue, les inhibiteurs de protéase ; puis, en 1998, une troisième famille, les inhibiteurs non nucléosidiques de la transcriptase inverse. L'association de ces molécules en trithérapies a conduit à une spectaculaire amélioration du pronostic des patients infectés par le VIH. Néanmoins, dès la fin des années 1990, certains patients étaient porteurs de virus résistants aux médicaments de ces trois familles, et peu d'options thérapeutiques alternatives existaient. Dans la plupart des publications décrivant cette situation d'impasse thérapeutique, les antirétroviraux avaient été prescrits
Université Pierre et Marie Curie (UPMC) Paris 6, Inserm UMR 5943, Assistance publique-hôpitaux de Paris (AP-HP), service des maladies infectieuses et tropicales, hôpital Pitié-Salpêtrière, 56, boulevard Vincent Auriol, 75013 Paris, France.

dominique.costagliola@ccde.chups.jussieu.fr

initialement sous la forme de mono- ou bithérapies à la majorité des patients [1-3].

Le projet PLATO II (Pursuing later treatment option $\mathrm{II}$ ), qui regroupe des populations de patients atteints du VIH dans le cadre du programme européen COHERE (Collaboration of observational HIV epidemiological research europe), s'est fixé pour objectif de caractériser les patients résistants aux trois premières familles d'antirétroviraux en Europe de 
l'Ouest entre 2000 et 2009. COHERE est un travail collaboratif sur des cohortes de patients infectés par le VIH en Europe et le projet PLATO II inclut les données de 91674 patients inclus dans 24 de ces cohortes. Il s'agit de patients infectés par le VIH qui ont débuté un traitement antirétroviral en 1998.

Cinq ans après l'instauration du premier traitement, le taux d'échec virologiquel était faible et estimé à $3,4 \%$ pour les trois premières familles de molécules [4]. Entre 2000 et 2005, au niveau collectif, l'incidence de l'échec virologique concernant les trois premières familles d'antirétroviraux a augmentée de 3,9 pour mille patients-années (intervalle de confiance [IC] à $95 \%=3,7$ à 4,1 ) à 8,8 pour mille patients-années (IC à $95 \%=8,5$ à 9,0$)$, puis a décru à 5,8 pour mille patients-années (IC à $95 \%=$ 5,6 à 6,1 ). La prévalence de l'échec virologique après traitement par les trois premières familles de molécules était de $0,3 \%$ (IC à $95 \%=0,27$ à 0,42) en 2000 . Elle a atteint 2,4\% (IC à $95 \%=2,24$ à 2,50) en 2005 , et est depuis restée stable, inférieure à $3 \%$ [5].

Au niveau individuel, 2476 patients avaient eu un échec virologique aux trois premières familles de molécules. La proportion de ceux dont la charge virale a pu être contrôlée par la modification de la combinaison antirétrovirale a augmenté de $19,5 \%$ en 2000 à $57,9 \%$ en 2009 ( $p<0,0001$ dans l'analyse multivariée). L'incidence du Sida (syndrome d'immunodéficience acquise) a décru de 7,7 pour 100 patients-années entre 2000 et 2002 , à 2,3 en 2008 et 1,2 en 2009 ( $p<0,0001$ dans l'analyse multivariée).

${ }^{1}$ L'échec virologique est défini dans cette étude comme la persistance d'une charge plasmatique virale supérieure à 500 copies/ml [6].
La mortalité a décru de 4,0 pour 100 patients-années entre 2000 et 2002 , à 1,9 en 2007 et 1,4 en 2008 ( $p=0,023$, analyse univariée), mais cette tendance n'était pas statistiquement significative après ajustement $(p=0,22)[6]$.

En Europe de l'Ouest, en pratique de routine, il y a donc eu une amélioration spectaculaire entre 2000 et 2009 du contrôle de la charge virale chez les patients qui répondaient mal (échec virologique) à l'administration des trois premières familles d'antirétroviraux. Cette amélioration est plus nette à partir de 2008 et s'est accompagnée d'une diminution de la morbidité. Si le premier résultat était attendu compte tenu des résultats des essais cliniques de nouveaux antirétroviraux chez les patients prétraités au cours de la période, les essais cliniques étaient de trop petite taille - chaque essai évaluant un nouvel antirétroviral - pour démontrer l'impact clinique qui a pu être mis en évidence grâce à cette collaboration de cohortes. Cet effet s'explique très probablement par la mise à disposition, au cours de la période de l'étude, de plusieurs antirétroviraux appartenant à de nouvelles familles, ou à des familles anciennes mais dans ce dernier cas présentant peu de résistance croisée avec les médicaments utilisés en première ou deuxième ligne. Le fait que ces médicaments, qui sont aussi utilisés chez les patients naïfs (non encore traités), soient plus faciles à prescrire et mieux tolérés expliquent sans doute également la diminution de l'incidence actuelle de l'échec virologique lors de traitements avec les molécules appartenant aux trois premières familles.

Ces résultats suggèrent l'impact de santé publique qu'a eu l'introduction de ces nouveaux médicaments. L'existence de nombreuses bases de données et cohortes cliniques de patients infectés par le VIH et pris en charge permet une évaluation post AMM (autorisation de mise sur le marché) à grande échelle des antirétroviraux. Il est important que ce modèle se développe pour d'autres maladies coûteuses ou fréquentes. Ces résultats montrent aussi que la mise à disposition de ces nouveaux médicaments à des prix abordables est urgente dans les pays du sud qui concentrent la grande majorité des personnes infectées par le VIH. $\diamond$

Impact of new antiretroviral therapy in HIV infected patients with virologic failure

\section{LIENS D'INTÉRÊT}

L'auteur déclare n'avoir aucun lien d'intérêt concernant les données publiées dans cet article.

\section{RéFÉRENCES}

1. Mocroft A, Phillips AN, Friis-Moller N, et al. Response to antiretroviral therapy among patients exposed to three classes of antiretrovirals: results from EuroSIDA study. Antiviral Therapy $2002 ; 7: 21-30$.

2. Ledergerber B, Lundgren JD, Walker AS, et al. Predictors of trend in CD4-positive T-cell count and mortality among HIV-1-infected individuals with virological failure to all three antiretroviral-drug classes. Lancet 2004 ; $364: 51-62$.

3. Costagliola D, Potard V, Duvivier C, et al. Impact of newly available drugs on clinical progression in patients with virological failure after exposure to three classes of antiretrovirals. Antiviral Therapy $2005 ; 10: 563-73$.

4. Lodwick L, Costagliola D, Reiss P, et al. Triple class virologic failure in HIV-infected patients on antiretroviral therapy for up to 10 years. Arch Intern Med $2010 ; 170: 410-9$.

5. Nakagawa F, Lodwick R, Costagliola D, et al. Calendar time trends in the incidence and prevalence of triple-class virologic failure in antiretroviral drug experienced people with HIV in Europe. J Acquir Immune Defic Syndr 2012 ; 59 : 294-9.

6. Costagliola D, Lodwick R, Ledergerber B, et al. Trends over calendar time in virological and clinical outcomes in individuals with HIV-1 infection and virologic failure of drugs from three antiretroviral drug classes: a cohort study. Lancet Infect Dis 2012 ; $12: 119-27$

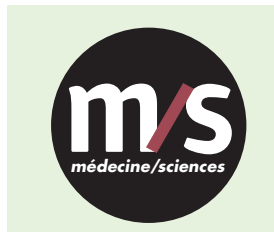

Tarifs d'abonnement $\mathrm{m} / \mathrm{s}-2012$

Abonnez-vous

à médecine/sciences
$>$ Grâce à $m / s$, vivez en direct les progrès des sciences biologiques et médicales

Bulletin d'abonnement page 790 dans ce numéro de $\mathrm{m} / \mathrm{s}$

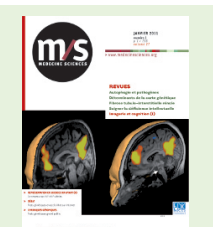

